\title{
Haar Wavelet Implementation to Various Partial Differential Equations
}

\author{
K.P. Mredula and D. C. Vakaskar
}

\begin{abstract}
The article brings together a series of algorithms with the modification in formulation of solution to various partial differential equations. The algorithms are modified with implementation of Haar Wavelet. Test examples are considered for validation with few cases. Salient features of multi resolution is closely compared with different resolutions. The approach combines well known finite difference and finite element method with wavelets. A detailed description of algorithm is attempted for simplification of the approach.
\end{abstract}

Index Terms-Multi Resolution; Haar Wavelet; Partial Differential Equations; Numerical Algorithms.

\section{INTRODUCTION}

Wavelet based numerical approaches have been investigated ever since the discovery of wavelet as a multi resolution tool to improve the accuracy and adaptive nature of wavelets. The mathematical property of multi resolution in wavelets motivates their use in solving partial differential equations. The most appealing feature of wavelet analysis in numerical solution of partial differential equation is the ability to estimate the local irregularities of the solution which allows self adaptive discretization with automatic mesh refinement [1]. They are studied and variedly used in partial differential equations of the category of modified burger equations, Schrodinger Poisson slater equation for study of system of electrons, Hartree Equation (in the field of relativity), Klein Gordaon Equation, Sine Gordon and perturbed NLS equations for modeling light bullets in two space dynamics [2].

Numerical algorithms in solving partial differential equations can be broadly classified into three categories 1) Core wavelet approach 2) Distinct wavelet approach 3) Multi resolution approach.

Core wavelet approach: Core wavelet approaches are called so as direct substitution of operators is done using wavelets. Solution is later obtained as a linear combination of wavelets and their coefficients. Improving the accuracy is done by increasing the resolution of wavelets. It is demonstrated in [6] for ordinary differential equations and in [7] for partial differential equations. The stability criteria is also stated in [7]. Both collocation and Galerikin approaches are included in core approaches. Galerikin approaches investigated in 90 s also formulate the solution in

Published on March 30, 2017.

K.P. Mredula is with the Sardar Vallabhbhai Patel Institute of Technology Vasad Gujarat India (e-mail: mredulakp.ash@svitvasad.ac.in).

D.C. Vakaskar with Department of Applied Mathematics Faculty of Technology and Engineering, The Maharaja Sayajirao University of Baroda Gujarat India (e-mail: dcvakaskar@gmil.com). form of wavelet basis and they discard the coefficients lesser than a threshold value.

Distinct wavelet approach: The approach is distinct as the wavelet bases evolve continuously in time and space and adapt to the flow properties as the solution evolves in time, for e.g. Lagrangian Wavelet Method [3]. The method served well for linear problems but failed due to wavelet collision during the extension of the approach to nonlinear problems as discussed in [4]. Most of the methods concentrate on time adaptively and formulate error reduction only in adapting to the problems in space. Time step is not considered as a control stability but most of space time wavelet methods uses different time adaptive strategies uses Compressible Euler equation for time adaptive method [5].

Multi resolution approach: In 1994 multi resolution approach was developed for solving hyperbolic conservative systems. It uses a hierarchical data which utilizes a very similar pattern as of multi scale nature of a disturbed flow. Wavelet basis represent the flow pattern and the wavelet coefficients are discarded for smooth areas which leads to compressed data. Hence the adaptive grid structure is developed which includes threshholding, of significant wavelets and it coefficients. Error is controlled as the grid adaptation error is controlled as a function of threshholding coefficients.

The study focuses on the implementation of all the approaches with consideration of Haar Wavelets as basis. Hence we describe the gradual development in the approaches which are ranging from finite difference, finite element, and linearization using Haar Wavelets.

We state the formulation of different algorithms with specific partial differential equation types. Section II deals with core approach on elliptic partial differential equation formulation with Haar Wavelets. Section III describes discontinuous Semi-Lagrangian approach on Vlasov equations. Section VI Numerical examples. Section V Conclusion and future scope.

\section{APPROACHES ON ELLIPTIC PARTIAL DIFFERENTIAL EQUATIONS}

The definition of Haar Wavelet and Multiresolution as in [7] are utilized. The approach includes the consideration of second order derivative as Haar approximations in both $\mathrm{x}$ and $\mathrm{y}$ direction.

$$
\begin{gathered}
w_{x x}=H^{T}(x) C_{1} H(y) \\
w_{y y}=H^{T}(x) C_{2} H(y) \\
w_{x x}+w_{y y}=0
\end{gathered}
$$

For the equation 
$C_{1}$ and $C_{2}$ represents the coefficients matrix of dimension $\mathrm{m}$. Integrating the equation (1) from 0 to $\mathrm{x}$ and 0 to $\mathrm{y}$ respectively twice we obtain,

$$
\begin{aligned}
& w_{x}(x, y)=H^{T}(x) \mathrm{Q}_{H}^{T} C_{1} H(y)+w_{x}(0, y) \\
& w(x, y)=H^{T}(x) \mathrm{Q}_{H}^{T} \mathrm{Q}_{H}^{T} C_{1} H(y)+x w_{x}(0, y)+\mathrm{w}(0, \mathrm{y})
\end{aligned}
$$

and

$$
\begin{aligned}
& w_{y}(x, y)=H^{T}(x) \mathrm{Q}_{H}^{T} C_{2} H(y)+w_{y}(\mathrm{x}, 0) \\
& w(x, y)=H^{T}(x) \mathrm{Q}_{H}^{T} \mathrm{Q}_{H}^{T} C_{2} H(y)+y w_{y}(\mathrm{x}, 0)+\mathrm{w}(\mathrm{x}, 0)
\end{aligned}
$$

The procedure includes assuming the highest derivative term as linear combination of Haar Wavelet, the successive integration is performed to obtain the original function. The terms are substituted into the governing equation to be solved which leads us to solve only system of algebraic equation in Haar coefficients.

\section{DisCONTINUOUS SEMI-LAGRANGIAN APPROACH}

Vlasov equation is widely implemented in plasma and astrophysics. The study of discontinuous Semi-Lagrangian Galerikin approach for Vlasov equations is motivated due to its utility. It describes the time evolution of the distribution function of plasma, consisting of the charged particles with long range interaction. Anatoly Vlasov in 1938 was first to suggest it for description of plasma then later it was discussed in monograph [9]. The Vlasov Poisson equation is given by

$\partial_{t} w(x, v, t)+\partial_{x}(v w(x, v, t))+\partial_{v}(E(x, t) w(x, v, t))=0, \quad x \in \Omega_{x}, v \in \Re$ $w(x, v, 0)=w_{0}(x, v)$

Where $w(x, v, t)$ is the phase-space distribution and $E(x, t)$ is the electric field obtained from the Poisson equation.

The Strang splitting is utilized to reduce the Vlasov eqution to one dimensional conservative form of equation given by

$$
\partial_{t} w(x, \mathrm{t})+\partial_{x}(c(x, t) w(x, \mathrm{t}))=0
$$

In Galerikin approach a weak formulation in each cell is solved considering test function $g \in G$ with $\mathrm{G}$ being the space of test function. By integrating equation over each cell

$$
I_{i}=\left[x_{i-\frac{1}{2}}, x_{i+\frac{1}{2}}\right]
$$

to obtain the form

$$
\int_{I_{i}} \partial_{t} w(x, \mathrm{t}) g(x) d x+\int_{I_{i}} \partial_{x}(c(x, t) w(x, \mathrm{t})) g(x) d x=0
$$

As the space derivative is applied on the test function the equation becomes

$$
\begin{aligned}
\int_{I_{i}} \partial_{t} w(x, \mathrm{t}) g(x) d x & -\int_{I_{i}} c(x, t) w(x, \mathrm{t}) \partial_{x} g(x) d x \\
+ & {[c(x, t) w(x, \mathrm{t}) g(x)]_{\substack{i+\frac{1}{2} \\
i+\frac{1}{2}}}^{x}=0 }
\end{aligned}
$$

The $L^{2}$ - projection can be considered as

$$
\begin{gathered}
w_{h}(x, t)=\sum_{i} \sum_{m=0}^{k} w_{m, i}(t) p_{m, i}(x) \\
\text { with basis of } P_{i}^{k} \quad \text { with, }\left\{p_{k, i}\right\}_{k=0, \ldots, K}
\end{gathered}
$$

This formulation is independent of the spatial order, which can be taken as a parameter.

For a two dimensional cell $T_{i, j}=U_{i} \times V_{j}$ is a tensor product of $U_{i}$ is in x direction and $V_{j}$ is in v direction where $V_{j}=\left[\mathrm{v}_{i-\frac{1}{2}}, \mathrm{v}_{i+\frac{1}{2}}\right]$ is in time direction. The Gauss Lobatto nodes are considered for $\mathrm{x}$ as $\mathrm{X}_{i_{g}}$ and for time it is $v_{j_{g}}$. Gauss weights are considered, always expressed for quadrature formula on the interval $[-1,1]$.

The subscript $\mathrm{n}$ is used to indicate time $t^{n}=n \Delta t$ and $w_{h}^{n}(x)=w_{h}\left(x, t^{n}\right)$. About the characteristics, we call $\mathrm{x}$ the variable at time $t^{n+1}$ and $x^{*}$ the foot of the characteristic at $t^{n}$. The formulation is considered as in [8]. The main idea is to discretize the equation (4), and to transform it into space integration using characteristics. This time integration leads to

$$
\begin{aligned}
\int_{U_{i}} w^{n+1}(x) g(x) d x & =\int_{U_{i}} w^{n}(x) g(x) d x+\int_{t^{n}}^{t^{n+1}} \int_{U_{i}} c w(x, t) g^{\prime}(x) d x d t \\
& -\int_{t^{n}}^{t^{n+1}}\left((c w(x, t) g(x))_{x_{i+\frac{1}{2}}^{-}}-(c w(x, t) g(x))_{x_{i-\frac{1}{2}}^{*}} d t\right.
\end{aligned}
$$

The terms on the right hand side are called $M_{0}, M_{1}$ and $M_{2}$

By integrating equation (2) over the domain ${ }^{\Omega}{ }^{-\frac{1}{2}}$ which is bounded by

$$
\begin{aligned}
& \left(x_{i-\frac{1}{2}}, t^{n+1}\right), \quad\left(x_{i-\frac{1}{2}}, t^{n}\right) \text { and }\left(x_{i-\frac{1}{2}}^{*}, t^{n}\right) \\
& \text { we obtain } \\
& \int_{\Omega_{i-\frac{1}{2}}}\left(\partial_{t} w(x, t)+\partial_{x}(c w(x, t))\right) \mathrm{dxdt}=0
\end{aligned}
$$

$$
\int_{\partial \Omega_{i-\frac{1}{2}}}\left(\partial_{t} w(x, t) \mathrm{n}_{t}+\partial_{x}(c w(x, t)) \mathrm{n}_{x}\right) \mathrm{d} s=0
$$

Using

$$
\int_{x_{i-\frac{1}{2}}^{*}}^{x_{i-\frac{1}{2}}} w\left(x, t^{n}\right) d x=\int_{t^{n}}^{t^{n+1}} c w\left(x_{i-\frac{1}{2}}, t\right) d t
$$


the expression become,

$$
M_{2}=-g\left(x_{i-\frac{1}{2}}\right) \int_{\substack{x_{i-\frac{1}{2}} \\ i-\frac{1}{2}}}^{x_{h}^{n}}(x) d x+g\left(x_{i+\frac{1}{2}}\right) \int_{\substack{x_{i+\frac{1}{2}}^{*} \\ i+\frac{1}{2}}}^{x_{h}^{n}}(x) d x
$$

For ${ }^{M_{1}}$ utilizing the Gaussian quadrature rule and the process of semi-lagrangian method we obtain

$$
\begin{aligned}
M_{1} & =\int_{t^{n}}^{t^{n+1}} \int_{U_{i}} c w_{h}(\mathrm{x}, \mathrm{t}) \mathrm{g}^{\prime}(\mathrm{x}) \mathrm{dxdt} \\
& =\int_{t^{n}}^{t^{n+1}} \frac{\Delta x_{i}}{2} \sum_{i_{g}} c w_{h}\left(x_{i_{g}}, t\right) g^{\prime}\left(x_{i_{g}}\right) \mathrm{w}_{i_{g}} d t \\
& =\frac{\Delta x_{i}}{2} \sum_{i_{g}} \int_{x_{i_{g}}^{*}}^{x_{i_{g}}} w_{h}^{n}(x) d x g^{\prime}\left(x_{i_{g}}\right) \mathrm{w}_{i_{g}}
\end{aligned}
$$

Foot of characteristics can be found by solving the ordinary differential equation

$$
\begin{aligned}
& \frac{d x}{d t}=c(x, t) \\
& x\left(t^{n+1}\right)=x_{i} \text { between } t^{n} \text { and } t^{n+1}
\end{aligned}
$$

Hence (4) becomes

$$
\begin{aligned}
& \int_{U_{i}} w_{h}^{n+1}(x) g(x) d x=\int_{U_{i}} w_{h}^{n}(x) g(x) d x \\
& +\Delta x_{i} \sum_{i_{g}} \int_{x_{i_{g}}^{*}}^{x_{i_{g}}} w_{h}^{n}(x) d x g^{\prime}\left(x_{i_{g}}\right) \\
& +\int_{x^{*} i_{-\frac{1}{2}}}^{x_{i}-\frac{1}{2}} w_{h}^{n}(x) d x g\left(x_{i_{-\frac{1}{2}}}\right) \\
& -\int_{\substack{x_{i} \\
+\frac{1}{2}}}^{x_{i}} w_{h}^{n}(x) d x g\left(x_{i_{+\frac{1}{2}}}\right)
\end{aligned}
$$

Choosing the function $\mathrm{g}$ as bases for $P_{i}^{k}$ the left term is $w_{k, i}^{n+1}$ and the first term of right hand side is $w_{k, i}^{n}$.

In two dimensional integration over the cell $M_{i, j}$ an additional integration in the orthogonal direction is required. Polynomials are only defined on a reference element $[-1,1]$ and all variables and functions taken on this reference element are indicated with a tilde.

The integration in $\mathrm{x}$ direction is

$$
\begin{gathered}
w_{k, M_{i, j}}^{n+1}=w_{k, M_{i, j}}^{n}+\frac{2}{\Delta x_{i}} \sum_{j_{g}} w_{j_{g}}\left(w_{i_{g}} \int_{x_{i_{g}}^{*}}^{x_{i g}} w_{h}^{n}\left(x, v_{j_{g}}\right) d x p_{\alpha_{k}}^{\prime}\left(x_{i_{g}}\right)\right) \mathrm{p}_{\beta_{k}}\left(v_{j_{g}}\right) \\
+\frac{2}{\Delta x_{i}} \sum_{j_{g}} w_{j_{g}}\left(\int_{x_{i-\frac{1}{2}}^{i-\frac{1}{2}}}^{x_{i}} w_{h}^{n}\left(x, v_{j_{g}}\right) d x p_{\alpha_{k}}\left(x_{i-\frac{1}{2}}\right)\right)- \\
\left.\int_{x_{i+\frac{1}{2}}^{*}}^{x_{i+\frac{1}{2}}} w_{h}^{n}\left(x, v_{j_{g}}\right) d x p_{\alpha_{k}}\left(x_{i+\frac{1}{2}}\right)\right) p_{\beta_{k}}\left(v_{j_{g}}\right)
\end{gathered}
$$

Here

$$
w_{k, M_{i, j}}^{n}=\frac{\Delta x_{i} \Delta v_{j}}{4} w_{k, M_{i, j}}^{n}
$$

Similar formulation in $\mathrm{v}$ direction could be done. Multi wavelet decomposition and reconstruction. Algorithm sequence

\section{NUMERICAL EXAMPLES}

The examples of $w_{x x}+w_{y y}=0$ is solved using the procedure discussed as in section II with

Case: 1

$w(x, 0)=2 x(1-x), \quad w(x, 1)=0 \quad$ and $\quad w(0, y)=0$

$w(1, \mathrm{y})=0$

Case :2

$$
\begin{aligned}
& w(x, 0)=x, \quad w(x, 1)=x^{2}-1 \quad \text { and } \quad w(0, y)=-y^{2} \\
& w(1, y)=-y^{2}
\end{aligned}
$$

Case 3:

$$
\begin{aligned}
& w(x, 0)=x^{2}, \quad w(x, 1)=x^{2}-1 \quad \text { and } \quad w(0, y)=-y^{2} \\
& w(1, y)=1-y^{2}
\end{aligned}
$$

Now for, Case 4 Solving Helmholtz equation $w_{x x}+w_{y y}=f(x, y) \quad$ with boundary conditions

$w(x, 0)=0, \quad w(x, 1)=0 \quad$ and $\quad w(0, y)=0$

$w(1, \mathrm{y})=0, \quad$ and $\mathrm{f}(\mathrm{x}, \mathrm{y})=\left(\mathrm{k}-2 \pi^{2}\right) \sin (\pi x) \sin (\pi y)$

with $\mathrm{k}=0.5$

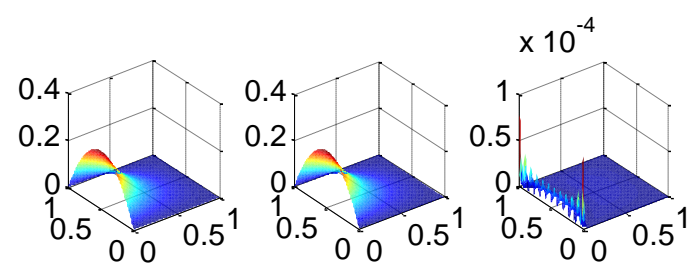

Fig 1. Comparison of finite difference solution, haar solution and error plot with 128 points, for case 1 


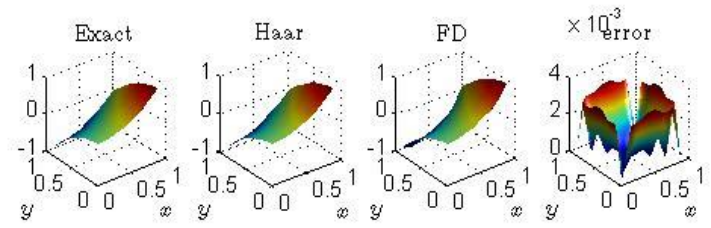

Fig 2. Comparison of exact, Haar, Finite difference and percentage error of Haar and exact solution. Case 3 with $\mathrm{m}=8$. Wavelet Haar method solution

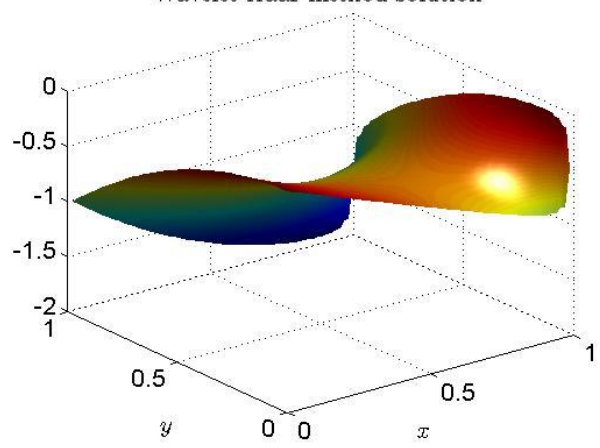

Fig 3. Solution of case 2 using haar wavelet method

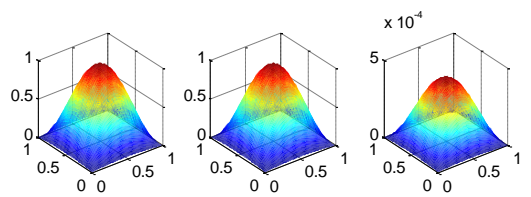

Fig 4. Comparison of the solution of helmohntz eqution with exact solution, haar and percentage error, with $\mathrm{m}=64$.

\section{CONCLUSION}

The paper reviews the formulation of algorithm for solving elliptic partial differential equation, states the semi lagrangian discontinuous approach and analysis few plots using the algorithm for elliptic partial differential equation. Different cases with boundary conditions were analyzed at different resolutions, which shows the significance of the approach used. It clearly indicates that a very fine change in resolution gives a better approximation. The plots as in fig 1 \& 2 compare the Haar approach with exact and finite difference method with the percentage error as low as 2 . Future work is to incorporate the multi resolution of wavelets to modify the already explored algorithms.

\section{REFERENCES}

[1] Schneider, K., \& Vasilyev, O. V. (2010). Wavelet Methods in Computational Fluid Dynamics*. Annual Review of Fluid Mechanics, 42(1), 473-503. http://doi.org/10.1146/annurev-fluid-121108-145637.

[2] Dong Xuanchun, "Numerical methods and their analysis for some nonlinear dispersion equations." Thesis 2012.

[3] Basdevant C Holshneider M Perrier V (1990). Travelling wavelets method. C.R. Acad Sci Ser I Math vol 310, pp.647-52.

[4] Berg J (2004). Wavelets in physics. Cambridge University Press.

[5] Domingues M. O. Gomes, s M Rousselo Schminder K. (2008) An adaptive multiresolution scheme with local time stepping for evolutionary partial differential equations. J Computational Physics vol 227, pp 3758-3780.

[6] Vakaskar, D. C., \& Mredula, K. P. . "Unified approach for solving initial valued and boundary valued ordinary differential equations using wavelet collocation method." 2013 Nirma University International Conference on Engineering (NUiCONE), 1-6. http://doi.org/10.1109/NUiCONE.2013.6780194. Ahmedabad Gujarat

[7] Mredula, K. P., \& Vakaskar, D. C. "Collocation method using wavelet for partial differential equation." Proceeding of International Conference on the occasion of silver Jubilee of Indian Society of Industrial and Applied Mathematics. (2016) Delhi India.

[8] Qiu, J. M., \& Shu, C. W. (2011). Positivity preserving semiLagrangian discontinuous Galerkin formulation: Theoretical analysis and application to the Vlasov-Poisson system. Journal of Computational Physics, 230(23), 8386-8409. http://doi.org/10.1016/j.jcp.2011.07.018.

[9] Vlasov equation. https://en.wikipedia.org/wiki/Vlasov_equation 